\title{
Mental Health Apps: Innovations, Risks and Ethical Considerations
}

\author{
Kyriaki G. Giota, George Kleftaras \\ Department of Special Education, University of Thessaly, Volos, Greece \\ Email: gkleftaras@uth.gr
}

Received 21 July 2014; revised 16 August 2014; accepted 25 August 2014

Copyright (C) 2014 by authors and Scientific Research Publishing Inc.

This work is licensed under the Creative Commons Attribution International License (CC BY). http://creativecommons.org/licenses/by/4.0/

cC) (7) Open Access

\begin{abstract}
The purpose of this article is to briefly review some of the innovations that mobile mental health apps present to consumers and mental health practitioners. Particular attention was given to understanding some of the important risks and the potential ethical dilemmas which may arise for counselors and psychologists who embrace them in their practice. Key considerations of issues pertinent to regulations, privacy concerns, and research are being discussed.
\end{abstract}

Keywords

Mental health, Apps, Counseling, Risks, Ethics

\section{Introduction}

Mobile technologies and networks are increasingly expanding in their sophistication and capacity, and new applications (software programs) are enhancing the ways in which individuals interact. Given the widespread use of smartphones and the increasing uptake of tablet devices, mHealth (the use of mobile and wireless technologies for health objectives) is believed to have the potential to improve the cost and quality of health care received, as well as reduce stigma and increase treatment accessibility globally [1]. Since the creation of Apple's iTunes App Store and Google's Android Market that allowed users to download mobile application software (apps) in 2008 [2], more and more sophisticated mobile apps are being developed in the field of mental health, and are being adopted almost as quickly as they can be developed [3]. The purpose of this paper is to review a number of innovations in the thriving department of mental health apps, as well as discuss important risks and potential ethical dilemmas which may arise for counselors and psychologists who would be willing to embrace them in their practice. 


\section{The Power of Mobile Technologies: Innovations in Mental Health}

Research shows that the majority of people and particularly young adults with mental health problems often do not seek professional help, despite the existence of effective psychological and pharmacological treatments [4]. The most common reasons include the lack of available services, especially in rural and remote areas, problems recognising symptoms, cost of treatment, time constraints and concerns about confidentiality and stigma [5]. However, mobile technologies, social networks and online media present an exciting, untapped opportunity for engaging younger populations in health and wellness activities, as well as promoting universal prevention and intervention [4] [6].

Mobile devices can become an ideal platform for self-monitoring symptoms and behaviours and providing personalized feedback, motivational support, and psychotherapeutic advice [1]. They can also be used for educational and training purposes (e.g. the APA Concise Dictionary [7]) or for administering standardized assessments and calculating scores (e.g. the PAR Assessment Toolkit [8]). Previous research suggests that mental health interventions delivered through mobile apps can have positive effects on a range of mental health problems, such as depression, stress, anxiety, and smoking cessation [9] [10]. These apps can be used as stand-alone self-help programs or in conjunction with guided programs offered via a website or through direct contact with a mental health professional. They can act as a bridge between therapy sessions and have the potential to improve adherence to treatment [11].

There is a growing amount of mental health apps for mobile devices that are youth-friendly, easily accessible and flexible to use. For example, Mobile Therapy [12] is an app where users can indicate their current mood at random times throughout the day, as well as record their energy levels, sleep patterns, activities, eating habits... The app offers therapeutic exercises ranging from breathing visualizations, to progressive muscle relaxation, to useful ways to disengage from a stressful situation. The information can later be charted and printed out so that users can evaluate the connection between their mood and other factors in their lives.

In the same sense, the CBT-based MyCompass [13] is a self-guided psychological treatment delivered via mobile phone and computer, designed to reduce mild-to-moderate depression, anxiety and stress, and improve work and social functioning. It encourages real-time self-monitoring of moods, mood triggers, and lifestyle behaviours using SMS text messaging and email prompts. Other apps include ReliefLink [14], a mood-tracking tool that tweets users regular affirmations, helps them make a safety plan and puts them it touch with nearby resources if they're contemplating suicide, and the Live Happy app [15] that is based on positive psychology and provides a set of daily activities scientifically proven to boost both short and long term happiness.

Even though the use of technology in mental health is relatively new, the idea of using games to help people with mental health problems is not novel. There are hundreds of mental health apps already on the market, and many incorporate elements of gamification (the use of video game techniques for more than entertainment purposes). Researchers have often focused on using these techniques with children, however, now it is common for adults to play games on their phones. For example Personal Zen [16] is an app based on a cognitive treatment for anxiety called attention-bias modification training. With the help of a game, people can learn to ignore threatening stimuli and focus on the good, feeling that way less anxious in stressful situations.

\section{Risks and Ethical Considerations}

However, mental health professionals should be aware of the risks, as well as potential ethical dilemmas when using mobile devices and applications for mental health problems. One of the most common issues revolve around the constraints of technology itself as a medium, for example problematic device and telecommunication technologies, battery failures, as well as unreliable or unstable internet connection [17]. Additionally, there is always the possibility of theft, loss, or malfunction of the mobile device that could have serious consequences when important data (e.g. electronic folders with history intakes, therapy session and billing notes, contact numbers and addresses of clients) are not stored in an easily accessible secondary location [3].

Many health and fitness applications often collect a large number of demographic and medical information by urging users to enter a lot of personally identifiable data, for example name, phone number, email address, age, gender, and photos [18]. They may also catalogue lifestyle information such as food consumption and exercise habits, or information related to their diagnoses, treatments and insurance (e.g. chronic health/mental health problems, screening results, medication dosages). Additionally, while using the app, people usually create a record of their daily routine and practices (e.g. diet, exercise, pregnancy and menstrual cycle, appointments and 
medication refills). Even if there is a privacy policy issued by the developer, there are, usually, no regulations that protect the privacy and security of personal health information, as once sensitive information is public via social media, users have little to no control over it [18].

Mental health professionals must take under consideration that free apps depend on advertising for funding and any information people provide to an app may be distributed to the developer, to third-party sites the developer may use for functionality reasons, and to unidentified third-party marketers and advertisers [19]. In the same sense, there is a strong possibility that they lack in reliable security, as they might transmit unencrypted personal data over insecure network connections, or allow ad networks to track users, that way raising serious concerns on their ability to protect the privacy and confidentiality of user information [19] [20].

Personal health information is of great value for cyber-criminals and can be used in order to obtain medical services and devices, or bill insurance companies for phantom services in the victim's name. As there are few legal protections, victims are forced to pay them or risk losing their insurance and/or ruining their credit ratings [21]. Fraudulent healthcare events can leave inaccurate data in medical records about tests, diagnoses and procedures that could greatly affect future healthcare and insurance coverage [22]. Furthermore, erroneous mental health information could potentially influence a person's social circle, school life or work opportunities [23].

Mental health professionals should carefully screen any apps that they are recommending and inform clients on whether their use could compromise their privacy [3] [4]. It is important to avoid applications that embed advertising or that seem to be primarily about selling products. User reviews, looking at the privacy policy in the developer's website, or learning about the app or the developer in the media, are ways to assess the quality and content of information of the app and validate the credibility of the app developer [18]. Some apps give users the option of trying out their features without entering personal information. Furthermore, deleting the app after no longer using it is an easy way to free up some memory, retain battery, as well as prevent the continuous broadcast of personal stored data. There are also apps that allow users to erase data in case the handheld device is lost or stolen. Another matter to consider is not to share confidential and personal health information through texts, as texting is not secure [18] [20]. Users should also be aware of the fact that smartphones and tablets can become ideal tracking tools, as people carry them almost everywhere they go, use them continuously, and rarely turn them off. An app might utilize various tracking technologies, such as collecting precise, real time location data or using geo-targeting (i.e., targeting by city and zip code) [24].

However, the most important issue that should be addressed is the lack of research evidence on the potential efficacy or effectiveness of mobile healthcare apps [1]. Research reviews show that the number of tested evidence-based mental health apps is small, and their studies usually have samples that are small, non-controlled, and non-randomised. Furthermore, few studies report findings of sustainable results for a period of more than three months, try to replicate these results or test the effects of these mobile interventions on everyday life, work and social functions in general [25] [26].

Although CBT interventions are successful in a number of mental health problems in face-to-face therapy [27], the evidence on the impact of CBT-based mHealth apps is still limited [28]. Few apps provide credible sources (e.g. systematic reviews, peer-reviewed articles) that allow users to locate information on the quality, accuracy and reliability of the content and outcomes [3] [25] [28]. Furthermore, there are apps that are criticized on their lack of effectiveness for the specific diagnostic and therapeutic recommendations that they claim to possess [29] [30]. It seems that the technology is advancing so fast that research is unable to keep up with every new development [31].

Only a small number of mHealth applications is designed by healthcare professionals and based on strong research, whereas a significant number of others are developed by institutions and individuals that are not mental health researchers or professionals [28]. It is of utmost importance to emphasize the fact that people download and use mobile apps at their own risk, as there currently are still no clear measures and certifications (from federal or other third-party institutions) in place to guarantee that the app a mental health professional is recommending to clients or downloading for personal use delivers credible content, contains safeguards for user data, and functions as described [2]. As apps become rapidly integrated into routine healthcare (e.g. logging data, checking and updating patient status), time seems be of the essence in creating strong guidelines for such technology, as well as certification programs for mHealth apps worldwide [32] [33].

\section{Conclusion}

Healthcare professionals have very little experience on how to best evaluate apps and older healthcare practitio- 
ners may be less inclined to use them or even be intimidated by these new technologies. Similarly, old clients may find it difficult to use and interpret the information provided to them by their smartphones [1]. We must take into consideration the fact that embracing new technologies cannot possibly replace the therapist-to-client relationship, but technology has the potential to provide better tools in making this more productive therapeutic alliance and enhancing the quality of care and support [28]. Future research should focus on certificating the quality and accuracy of mHealth apps, as well as creating courses that will train mental health professionals in using mobile technologies that could revolutionize approaches to patient care. Furthermore, psychologists, counsellors and therapists should contribute to the constantly growing body of evidence on the impact of mobile technology on mental health by reporting their experiences, publishing their findings, and improving their current practices [34].

\section{Acknowledgements}

The research has been co-financed by the European Union (European Social Fund-ESF) and Greek national funds through the Operational Program "Education and Lifelong Learning" of the National Strategic Reference Framework (NSRF)-Research Funding Program: Heracleitus II, investing in Knowledge Society through the European Social Fund.

\section{References}

[1] Becker, S., Miron-Shatz, T., Schumacher, N., Krocza, J., Diamantidis, C. and Albrecht, U.V. (2014) mHealth 2.0: Experiences, Possibilities, and Perspectives. JMIR mHealth uHealth, 2, e24. http://dx.doi.org/10.2196/mhealth.3328

[2] Vere, K.D. (2012) Tracking Growth: The iTunes App Store vs. Google Play. http://www.insidemobileapps.com/2012/09/26/tracking-growth-the-itunes-app-store-vs-google-play/

[3] Luxton, D.D., McCann, R.A., Bush, N.E., Mishkind, M.C., and Reger, G.M. (2011) mHealth for Mental Health: Integrating Smartphone Technology in Behavioral Healthcare. Professional Psychology: Research and Practice, 42, 505512. http://dx.doi.org/10.1037/a0024485

[4] Kazdin, A.E. and Rabbitt, S.M. (2013) Novel Models for Delivering Mental Health Services and Reducing the Burdens of Mental Illness. Clinical Psychological Science, 1, 170-191. http://dx.doi.org/10.1177/2167702612463566

[5] Gulliver, A., Griffiths, K. and Christensen, H. (2010) Perceived Barriers and Facilitators to Mental Health Help Seeking in Young People: A Systematic Review. BMC Psychiatry, 10, 113.

http://dx.doi.org/10.1186/1471-244X-10-113

[6] Whittaker, R.A., Merry, S., Stasiak, K., McDowell, H., Doherty, I., Shepherd, M., Dorey, E., Ameratunga, S. and Rodgers, A. (2012) Universal Depression Prevention via Mobile Phones. Journal of Mobile Technology in Medicine, 1, 4S. http://dx.doi.org/10.7309/jmtm.28

[7] American Psychological Association (2012) APA Concise Dictionary. Mobile Application Software. https://play.google.com/store/apps/details?id=com.apa.cdp.full

[8] PAR, Inc. (2014) PAR Assessment Toolkit. Mobile Application Software. https://itunes.apple.com/us/app/par-assessment-toolkit/id356919305?mt=8

[9] Harrison, V., Proudfoot, J., Wee, P.P., Parker, G., Pavlovic, D.H. and Manicavasagar, V. (2011) Mobile Mental Health: Review of the Emerging Field and Proof of Concept Study. Journal of Mental Health, 20, 09-524. http://dx.doi.org/10.3109/09638237.2011.608746

[10] Whittaker, R., McRobbie, H., Bullen, C., Borland, R., Rodgers, A. and Gu, Y. (2012) Mobile Phone-Based Interntions for Smoking Cessation. Cochrane Database of Systematic Reviews, 11, 06611. http://dx.doi.org/10.1002/14651858

[11] Proudfoot, J. (2013) The Future Is in Our Hands: The Role of Mobile Phones in the Prevention and Management of Mental Disorders. Australian \& New Zealand Journal of Psychiatry, 47, 111-113. http://dx.doi.org/10.1177/0004867412471441

[12] Morris, M.E., Kathawala, Q., Leen, T.K., Gorenstein, E.E., Guilak, F., Labhard, M. and Deleeuw, W. (2010) Mobile Therapy: Case Study Evaluations of a Cell Phone Application for Emotional Self-Awareness. Journal of Medical Internet Research, 12, e10. http://dx.doi.org/10.2196/jmir.1371

[13] Proudfoot, J., Clarke, J., Birch, M.R., Whitton, A.E., Parker, G., Manicavasagar, V., et al. (2013) Impact of a Mobile Phone and Web Program on Symptom and Functional Outcomes for People with Mild-to-Moderate Depression, Anxiety and Stress: A Randomised Controlled Trial. BMC Psychiatry, 13, 312. http://dx.doi.org/10.1186/1471-244X-13-312 
[14] Woodruff Health Sciences Center (2013) Suicide Prevention App Awarded \$50,000 Prize at White House Conference. http://news.emory.edu/stories/2013/09/kaslow_relieflink_app/campus.html

[15] (2009) Happiness Is an iPhone App. University of California, Riverside. http://newsroom.ucr.edu/news_item.html?action=page\&id=2139

[16] Dennis, T.A. and O’Toole, L.J. (2014) Mental Health on the Go. Effects of a Gamified Attention-Bias Modification Mobile Application in Trait-Anxious Adults. Clinical Psychological Science, Published Online. http://dx.doi.org/10.1177/2167702614522228

[17] Burns, M.N., Begale, M., Duffecy, J., Gergle, D., Karr, C.J., Giangrande, E., et al. (2011) Harnessing Context Sensing to Develop a Mobile Intervention for Depression. Journal of Medical Internet Research, 13, e55. http://dx.doi.org/10.2196/jmir.1838

[18] Ackerman, L. (2013) Mobile Health and Fitness Applications and Information Privacy: Report to California Consumer Protection Foundation. Privacy Rights Clearinghouse. https://www.privacyrights.org/mobile-health-and-fitness-apps-what-are-privacy-risks

[19] Harris, K.D. (2013) Privacy on the Go: Recommendations for the Mobile Ecosystem. California Department of Justice. http://oag.ca.gov/sites/all/files/agweb/pdfs/privacy/privacy_on_the_go.pdf

[20] Njie, C.M.L. (2013) Technical Analysis of the Data Practices and Privacy Risks of 43 Popular Mobile Health and Fitness Applications. Privacy Rights Clearinghouse. https://www.privacyrights.org/mobile-medical-apps-privacy-technologist-research-report.pdf

[21] Dolan, P.L. (2013) Health Data Breaches Usually Aren’t Accidents Anymore. http://www.amednews.com/article/20130729/business/130729953/4 /

[22] Dolan, P.L. (2013) Data Breach Insurance Goes Mainstream in Health Care. http://www.amednews.com/article/20130819/business/130819963/2/

[23] Wakefield, J.C. (2007) The Concept of Mental Disorder: Diagnostic Implications of the Harmful Dysfunction Analysis. World Psychiatry, 6, 149-156. http://www.ncbi.nlm.nih.gov/pmc/articles/PMC2174594/

[24] Caetano, L. (2013) Location, Location, Location: Three Reasons It Matters for Your Smartphone. https://blogs.mcafee.com/consumer/mobile-security/location-location-location-three-reasons-it-matters-for-your-smart phone

[25] Fiordelli, M., Diviani, N. and Schulz, P.J. (2013) Mapping mHealth Research: A Decade of Evolution. Journal of Medical Internet Research, 15, e95. http://www.jmir.org/2013/5/e95/ http://dx.doi.org/10.2196/jmir.2430

[26] Donker, T., Petrie, K., Proudfoot, J., Clarke, J., Birch, M.R. and Christensen, H. (2013) Smartphones for Smarter Delivery of Mental Health Programs: A Systematic Review. Journal of Medical Internet Research, 15, e247. http://dx.doi.org/10.2196/jmir.2791

[27] Hofmann, S.G., Asnaani, A., Vonk, I.J.J., Sawyer, A.T. and Fang, A. (2012) The Efficacy of Cognitive Behavioral Therapy: A Review of Meta-Analyses. Cognitive Therapy and Research, 36, 427-440. http://dx.doi.org/10.1007/s10608-012-9476-1

[28] Aguilera, A. and Muench, F. (2012) There’s an App for That: Information Technology Applications for Cognitive Behavioral Practitioners. The Behavior Therapist, 35, 65-73. http://www.abct.org/docs/PastIssue/35n4.pdf

[29] Buijink, A.W.G., Visser, B.J. and Marshall, L. (2012) Medical Apps for Smartphones: Lack of Evidence Undermines Quality and Safety. Evidence-Based Medicine, 18, 90-92. http://dx.doi.org/10.1136/eb-2012-100885

[30] Wolf, J.A., Moreau, J.F., Akilov, O., Patton, T., English, III., J.C. Ho, J. and Ferris, L.K. (2013) Diagnostic Inaccuracy of Smartphone Applications for Melanoma Detection. JAMA Dermatology, 149, 422-426. http://dx.doi.org/10.1001/jamadermatol.2013.2382

[31] Riley, W.T., Rivera, D.E., Atienza, A.A., Nilsen, W., Allison, S.M. and Mermelstein, R. (2011) Health Behavior Models in the Age of Mobile Interventions: Are Our Theories Up to the Task? Translational Behavioral Medicine, 1, 53-71. http://dx.doi.org/10.1007/s13142-011-0021-7

[32] Ozdalga, E., Ozdalga, A. and Ahuja, N. (2012) The Smarphone in Medicine: A Review of Current and Potential Use among Physicians and Students. Journal of Medical Internet Research, 14, e128. http://dx.doi.org/10.2196/jmir.1994

[33] Tomlinson, M., Rotheram-Borus, M.J., Swartz, L. and Tsai, A.C. (2013) Scaling Up mHealth: Where Is the Evidence? PLOS MEDICINE, 10, e1001382. http://dx.doi.org/10.1371/journal.pmed.1001382

[34] Giota, K.G. and Kleftaras, G. (2014) Cyberpsychology and Social Media: Online Social Support in Mental Health. In: Kommers, P., Isaias, P. and Issa, T., Eds., Perspectives on Social Media: A Yearbook, Routledge, London. 
Scientific Research Publishing (SCIRP) is one of the largest Open Access journal publishers. It is currently publishing more than 200 open access, online, peer-reviewed journals covering a wide range of academic disciplines. SCIRP serves the worldwide academic communities and contributes to the progress and application of science with its publication.

Other selected journals from SCIRP are listed as below. Submit your manuscript to us via either submit@scirp.org or Online Submission Portal.
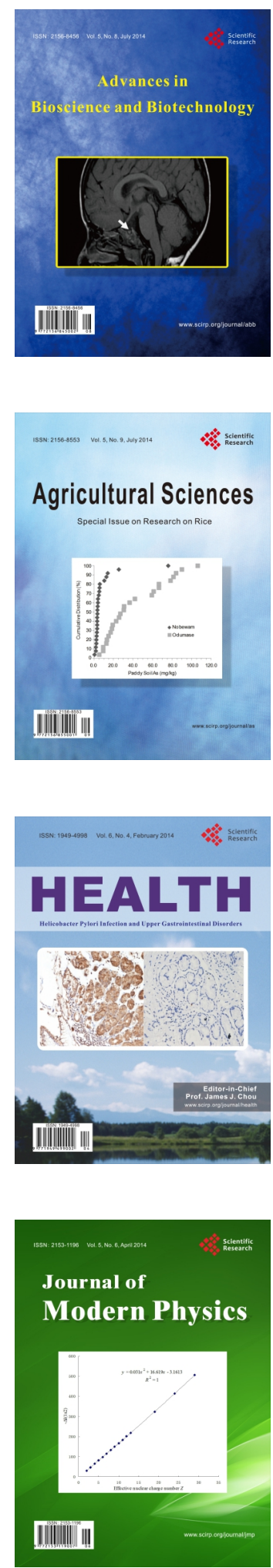
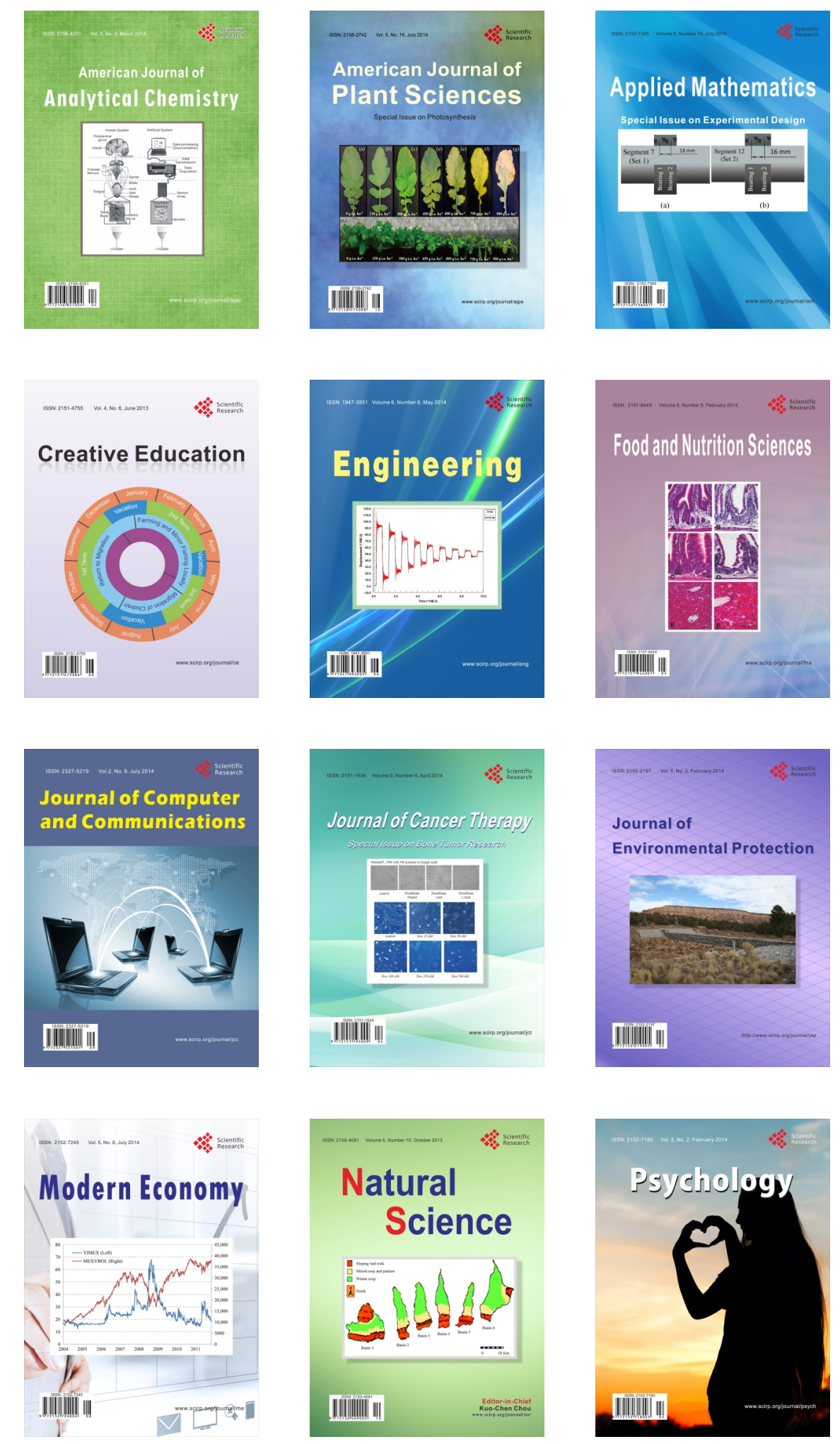